\title{
Presenting Walking Route for VR Zombie
}

\author{
Nobuchika SAKATA $^{\dagger a}$, Member, Kohei KANAMORI ${ }^{\dagger \dagger}$, Tomu TOMINAGA ${ }^{\dagger \dagger}$, Nonmembers, \\ Yoshinori HIJIKATA $^{\dagger \dagger}$, Member, Kensuke HARADA ${ }^{\dagger \dagger}$, and Kiyoshi KIYOKAWA ${ }^{\dagger}$, Nonmembers
}

\begin{abstract}
SUMMARY The aim of this study is to calculate optimal walking routes in real space for users partaking in immersive virtual reality (VR) games without compromising their immersion. To this end, we propose a navigation system to automatically determine the route to be taken by a VR user to avoid collisions with surrounding obstacles. The proposed method is evaluated by simulating a real environment. It is verified to be capable of calculating and displaying walking routes to safely guide users to their destinations without compromising their VR immersion. In addition, while walking in real space while experiencing VR content, users can choose between 6-DoF (six degrees of freedom) and 3-DoF (three degrees of freedom). However, we expect users to prefer 3-DoF conditions, as they tend to walk longer while using VR content. In dynamic situations, when two pedestrians are added to a designated computer-generated real environment, it is necessary to calculate the walking route using moving body prediction and display the moving body in virtual space to preserve immersion.
\end{abstract}

key words: virtual reality, walking assist, degree of freedom, VR zombie

\section{Introduction}

In recent years, highly immersive virtual reality (VR) environments have become popular due to the increased availability of affordable head-mounted displays (HMDs) among general consumers. Advancements in inside-out tracking and wireless technology have enabled users to experience VR while freely walking within an indoor environment, instead of merely sitting in a chair and looking over a 360degree virtual environment. Currently, a significant proportion of road accidents are caused by the use of mobile devices by drivers or pedestrians - the so-called "Smartphone Zombies". However, HMDs obscure their users' entire fields of view, to a much greater extent than other portable devices like smartphones. Therefore, when VR is applied to outdoor activities such as walking in the future, it is even more likely to induce dangerous accidents. Henceforth, we refer to a user in such a scenario as a "VR Zombie". To avoid collision of VR zombies with obstacles, it is necessary to present information about the ambient real space to the user within the VR interface. However, the method of

Manuscript received April 10, 2020.

Manuscript revised August 21, 2020.

Manuscript publicized September 30, 2020.

${ }^{\dagger}$ The authors are with affiliated to Nara Institute of Science and Technology, Ikoma-shi, 630-0192 Japan.

${ }^{\dagger}$ The authors are with affiliated to Osaka University, Toyonakashi, 560-8531 Japan.

${ }^{\dagger \dagger}$ The author is with affiliated to Kwansei Gakuin University, Nishinomiya-shi, 662-8501 Japan.

a)E-mail: sakata@is.naist.jp

DOI: 10.1587/transinf.2020EDP7084 presentation may compromise the user's immersion, which is an important element of VR. It seems that users do not always use some functions when using mobile devices while walking, which might impair the quality of the VR experience. Therefore, in this study, we propose a system to guide VR users to their destinations in real space while simultaneously avoiding collisions with obstacles and preserving their immersion.

By investigating existing walking assistance techniques in real space for VR users, Kanamori et al. concluded that users can be guided to walk in real space without compromising their immersion by presenting information about real-space obstacles to them in terms of VR objects that harmonize with the surrounding virtual environment in the form of appropriate clues scattered within the play area [1], [2]. This aids the user to imagine the actual shapes in the real space based on the virtual clues, and thereby, avoid collisions with them. However, even in this case, the user needs to be conscious of the ambient real space, and so complete immersion is not achieved. Further, Kanamori et al. [1] assumed the user to be partially aware of the realspace information surrounding the play area. This makes such a method difficult to apply in arbitrary situations, other than in pre-designated spots. Therefore, it is necessary to develop a method which enables users to use routes based on real-space information displayed within the virtual space that does not compromise their immersion. We achieve this by proposing a system that detects the positions of obstacles in the real-world surroundings of the user, automatically calculates the optimal walking route, and presents it to the user (Fig. 1). The user is not required to be aware of any realspace information beforehand to apply this technique, and can reach the destination simply by walking along the indicated route.

While guiding VR users towards their destinations in real space, it is necessary to ascertain the degrees to which users' movements in real space can be reflected within the virtual space to design a comfortable VR experience. For example, within the interface of VR with 6 DoFs (six degrees of freedom), the avatar of a user in the virtual space may move in an unintended direction corresponding to the user's movements in real space as the coordinate movement of walking is directly reflected in the virtual space in such an environment. On the other hand, in the case of a 3DoF (three degrees of freedom) VR environment, the aforementioned problem does not occur as the coordinate move- 


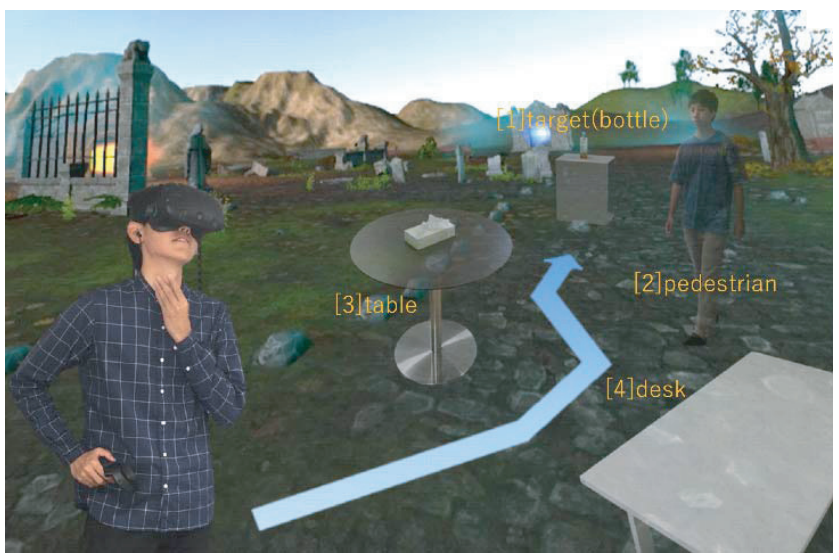

Fig. 1 Concept of the proposed system. Numbers 1-4 represent realspace objects functioningas obstacles, but the HMD user cannot see them within theVR interface. Only the walking path (highlighted by the blue arrow and line) is presented to the user in the proposed system.

ment of walking is not reflected on the avatar in the virtual space in such a scenario. However, this introduces the possibility of compromised immersion due to the incongruence between physical and virtual movements during walking [3], [4].

In this study, we evaluate the proposed walking assistance technique by calculating the optimal walking routes for users based on their preferred DoF conditions. However, evaluation of the method in real space would entail possible collisions of the subject of the experiment with neighboring pedestrians or obstacles. Further, ensuring the consistent movement of actual pedestrians in real space is also difficult. Thus, in order to guarantee the security of the subject and maintain consistent experimental conditions, we conducted the evaluation experiments by simulating a real environment within a virtual environment. The results confirm the effectiveness of the proposed method and identify the optimal DoF condition.

\section{Related Works}

Several studies have proposed methods to display an image of the user's body within the virtual space using augmented virtuality. Steinicke et al. realized the display of a user's hands in virtual space using a chroma key and improved presence in the virtual space [5]. Tecchia et al. developed an MR system that enables users to interact with virtual objects by measuring the positional relationships between their hands in real space using Kinect [6]. McGill et al. conducted a questionnaire survey about the user-friendliness of VR-HMDs among 108 VR-HMD users [7]. The results demonstrated that a significant proportion of people desire improved interaction with the real space while wearing VR HMDs. In addition, the study revealed that users are unable to perceive their surroundings while wearing HMDs and find it cumbersome to remove the HMD for every interaction. The authors conducted another study to resolve these problems and obtained the following conclusions. Firstly, the partial display of real space within a virtual environment is less harmful to users' immersion than the need to remove HMDs to interact with reality. Secondly, the automatic display of real-space information is less harmful to immersion than intentional display initiated by the user. Thirdly, virtual projection of a person in the real-space vicinity of the user within the VR environment does not affect immersion. Further, the subjects opined that the appearances of such projected real-space objects should be modified to harmonize them with the ambient VR content. Budhiraja et al. investigated a display technique using peripheral real space with respect to a variety of VR content [8]. They reported that most subjects favor only their hands and the objects of interest to be displayed, with only the boundaries of the surrounding objects being visible. The aforementioned studies suggest that users find it difficult to interact with real space while wearing a VR-HMD and the selective display of realspace information within the virtual space can resolve this problem. However, these methods primarily focus on static users, and therefore, cannot be utilized in the case of realspace interactions involving walking.

The enhancement of immersion within the virtual environment is crucial to free movement in the virtual space. Several methods have been proposed to enable users to move within the virtual space [9]-[11], but actually walking in real space remains the most natural and effective method [9], [12], [13]. Sing et al. presented the features of ambient real spaces to VR-users via real-time voxel-themed three-dimensional reconstruction. In addition, they developed walking assistance techniques for users to avoid obstacles while remaining immersed in the virtual environment [14]. Several studies have been conducted on spatial perception in virtual spaces. It has been demonstrated that users underestimate distances in virtual environments compared to real environments [15], [16], and this effect is particularly pronounced when the user is using an HMD [17][19]. Further, user perception in a virtual environment depends on the size and shape of the user's avatar [20], [21]. Thus, it is evident that spatial perception is significantly different in real and virtual environments and it is unclear whether the distance information pertaining to real-world objects displayed to VR-users within virtual environments is accurately perceived.

In the context of navigation aided by smartphones and HMDs, Rahimian et al. investigated the effectiveness of using smartphones to alert pedestrians of approaching vehicles [22]. As in the case of this study, they also conducted experiments in a computer-generated real environment to guarantee the safety of the subjects and to accurately measure relevant data, such as the direction of the user's gaze. The authors guided users to their real-world destinations using visual information about the real environment obscured by HMDs. They concluded that experiments in simulated real environments are reasonable for the evaluation of walking assistance techniques. Several studies have proposed methods to address the constraints of real spaces within virtual environments by assuming the real space to 
be the main information queue, superimposing virtual information on the real environment via HMDs, and comparing the achieved navigation efficiency to those of handheld displays [23], [24]. However, till date, no studies have investigated real environment navigation during the use of VR by regarding the virtual space as the main information queue.

The influence of DoFs in head tracking on VR users has also been extensively researched. Buttussi et al. experimented on subjects experiencing scenario-based VR via three different display conditions (desktop monitor, 3-DoF narrow field of view HMD, and 6-DoF wide field of view HMD) [25]. This experiment did not reveal any significant difference between the users' understandings of the scenario via the three types of displays. However, even though no statistically significant difference in immersion in the virtual space was observed, the immersion was observed to be higher under high-fidelity 6-DoF conditions. Pal et al. compared the user's performances under three headtracking conditions (6-DoF, Rotation 3-DoF, and Translation 3-DoF) [26]. The results demonstrated that the translation 3-DoF condition produced noticeably poor performance. However, no significant difference was noticed between the performances under the 6-DoF and rotation 3-DoF conditions. However, till date, no studies have investigated the optimal DoF conditions for VR-users walking in real space.

\section{Proposed Method}

The aim of this study is to develop a navigation system that is capable of automatically calculating the optimal route to be followed by a user while walking to avoid collisions with surrounding obstacles. To this end, the proposed system recognizes surrounding real-space objects in real time using sensors attached to HMDs or the external real environment. In this study, we begin by verifying the effectiveness of displaying the walking route within virtual space to support walking in real space. To guarantee the safety of the subjects of the experiment and to eliminate dependence of the performance of the proposed system on the accuracy of the real space sensing, we evaluate it in a computergenerated real environment. In other words, the reason for preparing a computer-generated real environment is to control pedestrian movement between subjects and provide an atmosphere of walking in the city in a safe real environment. It is assumed that the real environment of a user wearing the HMD can be perfectly detected. In the computer-generated real environment, roads and obstacles are reproduced to imitate real-world outdoor situations (Fig. 2, upper left). In addition, video see-through HMDs and AR glasses can be used to show users an AR environment with obstacles superimposed on the real world. However, this was not adopted for the following reasons. When an AR object is superimposed on real-world images captured by a front camera of HMD and displayed on the HMD, there is a time delay as compared to displaying computer-generated real environments on the HMD. Moreover, if a lot of CGs of obstacles

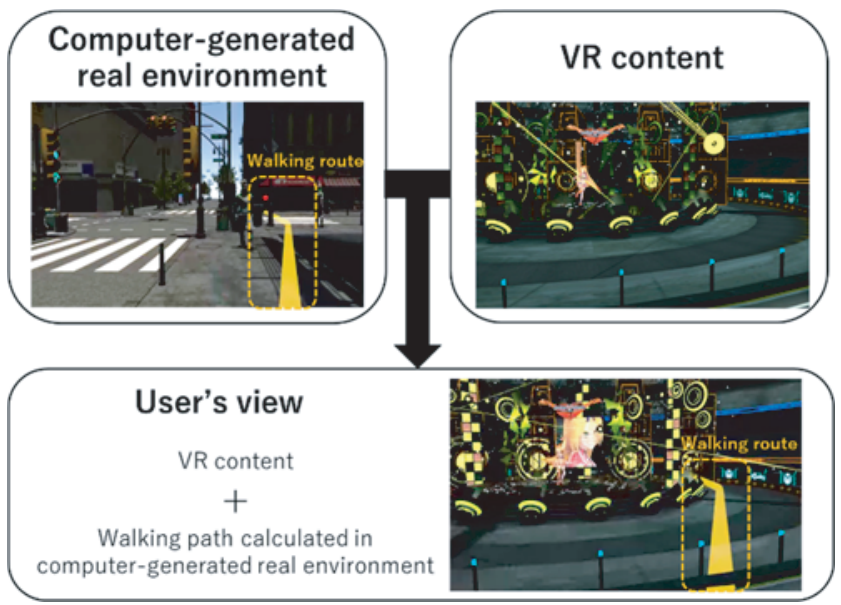

Fig. 2 The user's perspective is composed of the optimal walking route obtained via NavMesh and the VR content.

and town environment are superimposed on a video of the real world where there are no obstacles, it will be the same as the case of VR (computer-generated real environments). Because 3 of these reasons, we adopted a method of displaying computer-generated real environment on VR-HMD as an imitation of walking in the real world.

To implement this system, we used the VIVE Pro (765 g, binocular resolution: $2880 \times 1600$ ) as an HMD and Unity, which is a $3 \mathrm{D}$ game engine, to simulate the real environment.

In this study, we conduct experiments using a computer-generated real environment wherein it is assumed that the real environment of the user is perfectly detected. We use NavMesh to calculate the optimal walking route in the simulated real environment, which is a route search function in Unity used to define a start position and a destination, by searching for neighboring polygons via the $\mathrm{A}^{*}$ algorithm, and calculating the shortest route to the destination. In addition, in this study, only the navigation part is the subject of research. As for the destination input, we assumed that it was set to be completed before the navigation started. In actual use, it is assumed that the destination is entered with the smartphone before the HMD is attached to a user or entered with the special software such as Google map. Furthermore, we do not consider that the final destination may change during navigation.

\subsection{Computer-Generated Real Environment and VR Con- tent}

In this study, we assumed the experimental scenario to be one in which the user wears an immersive HMD and experiences VR content while walking toward his destination in real space. In this section, we discuss the computergenerated real environment and the VR content used in the experiment. 


\subsubsection{Computer-Generated Real Environment}

VR is expected to be implemented in various real environments in the near future based on inside-out head tracking systems and wireless HMDs and controllers. Therefore, in our experiment, we assumed a real environment in which a "Smartphone Zombie" uses a smartphone while walking down a sidewalk (Figs. 3 and 6). The sidewalk was assumed to be adjacent to a street, on which vehicles ply at regular intervals. This outdoor situation was simulated as a computer generated scenario to invoke the psychological dread of a dangerous situation in the subject of the experiment while ensuring his physical safety.

When virtual pedestrians were included in the computer-generated scenario, the calculated optimal walking route was observed to fluctuate in real time. While the subjects were in the process of walking along the fluctuating route, the displacements of the user's walking trajectory could not be accurately estimated. Even if the virtual pedestrians walked along the same route, the closest timings corresponding to distinct subjects exhibited slight differences, inducing drastic differences in the indicated route. If the indicated walking route is dependent on the characteristics of other virtual pedestrians, the length of the optimal walking trajectory would be different for each subject. To avoid this, we first conducted an experiment assuming that no pedestrians exist near the subject. However, even in this scenario, the subjects did not follow the same trajectory at all times. As the route from the subject's current coordinate to the destination is constantly recalculated, the route fluctuates in real time based on changes in the subject's coordinates caused by small movements by the subject. In short, the walking route is not completely fixed in the virtual space, but is slightly altered by the subjects' behaviors and preferences. Finally, by observing and evaluating the slightly changed values, the preferences of each subject can be ascertained corresponding to each DoF.

We position this experiment, in a setting where there are no pedestrians or moving cars, as an ideal environment for VR Zombie. If participants as VR Zombie cannot immerse themselves in VR content or reach their destination in this ideal state, then adding moving cars and pedestrians to the experience would be pointless. Therefore, Therefore, the result of this experience in the ideal state is a type of max performance of our proposed system.

\subsubsection{VR Content}

While various kinds of VR content exist, we opine that certain categories of VR that involve frequent interactions and multi-directional events, such as First Person Shooter games, should not be used during walking, as such content involves too many obstacles and significantly affects the direction of the user's gaze. People are known to watch music videos while walking on streets and trains. Therefore, in our experiment, we adopted a specific live music content for
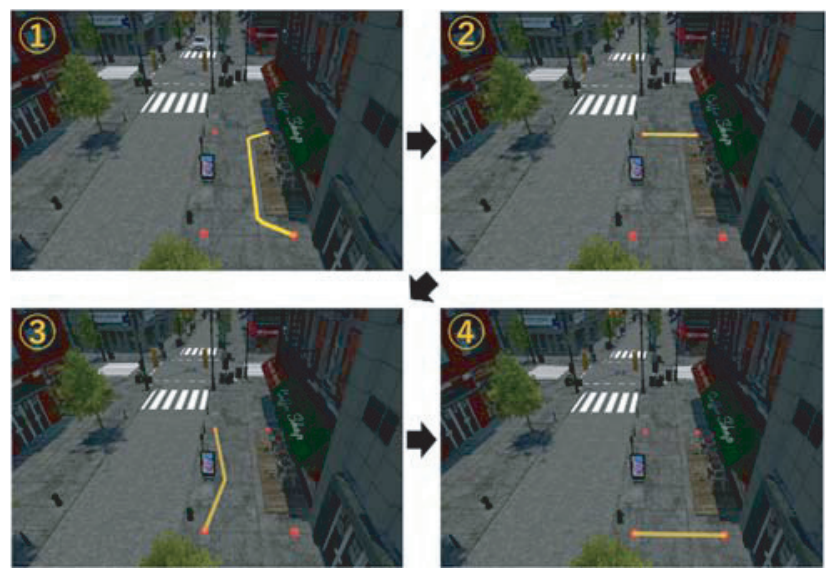

Fig. 3 Fluctuating walking route in a computer-generated real environment.

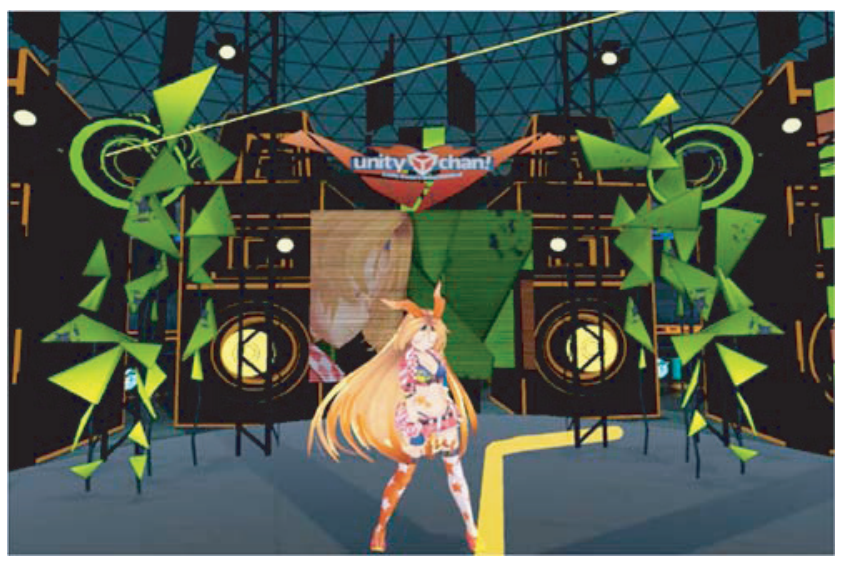

Fig. 4 Image of VR live content and the optimal walking route projected via the HMD.

the subjects to watch as the VR content. The content was provided by Unity (Fig. 4).

\section{DoF Experiment}

In this study, we conducted the experiment assuming a situation where a user wears an immersive HMD and experiences live VR music content while walking toward a destination in real space. We attempted to answer the following two research questions (RQs):

RQ1) Can the user be safely guided to his destination in real space without compromising his immersion by displaying solely the optimal walking route to the destination within the VR environment?

RQ2) Which degree of freedom is optimal to track the movements of the user's head with respect to the VR content to ensure that the user can enjoy the VR content while walking toward the destination in real space? 

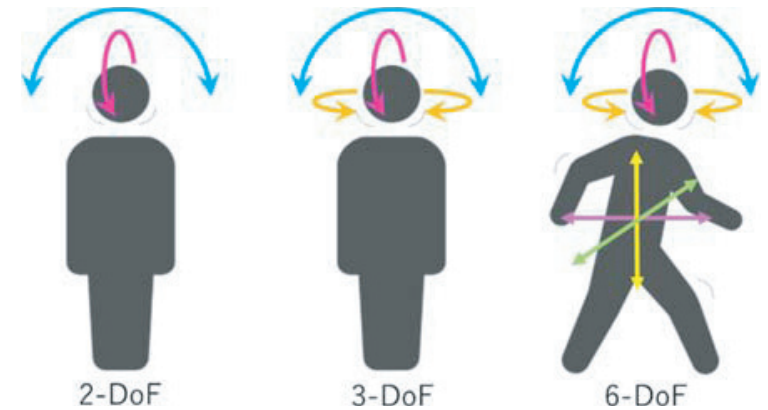

Fig. 5 DoFs in head tracking to reflect VR content.

\subsection{Experimental Design}

In this experiment, we guided the subjects towards predefined destinations within the designated computer-generated real environment by displaying solely the optimal walking route within the VR environment, as depicted in Fig. 2. In order to determine the characteristics of the computergenerated real environment necessary for the users and ensure the reliability of the calculated walking route, subjects were asked to walk along the presented route in the absence of any VR content before conducting the actual experiment. In addition, we allowed the subjects to remain stationary and experience the VR content before the actual experiment. Then, the subjects were asked to answer a questionnaire about their VR immersion while being stationary. The results of the questionnaire were taken to be a reference of VR experience under normal conditions (watching VR live content without any movement). Following this, each subject was asked to perform the experimental tasks under the following three conditions.

1) 2-DoF: pitch and roll rotation are reflected to view angle of participant (Fig. 5, left).

The VR avatar can be always kept at the center of the field of view, because the yaw is not reflected in view angle

2) 3-DoF: pitch, roll and yaw rotation are reflected in the view angle of the participant with the gaze direction reset function. (Fig. 5, center).

The participant can watch the VR avatar from various angles based on head posture but the participant cannot be close to the VR avatar. In the case where the VR avatar cannot watch in the participant's field of view along with the head posture, the participant can reset the view angle to the initial angle by grabbed controller.

3) 6-DoF: rotation 3-DoF and translation 3-DoF are reflected to view angle of participant with viewpoint reset function (Fig. 5, right)

Participants can watch VR avatars from various angles based on head posture and the participant can be close to VR avatar. In the case where the VR avatar cannot watch in the participant's field of view along with the participant's movement, participant can reset the viewpoint to an initial point using a grabbed controller. At the initial point, the VR avatar can be watched at the center of the

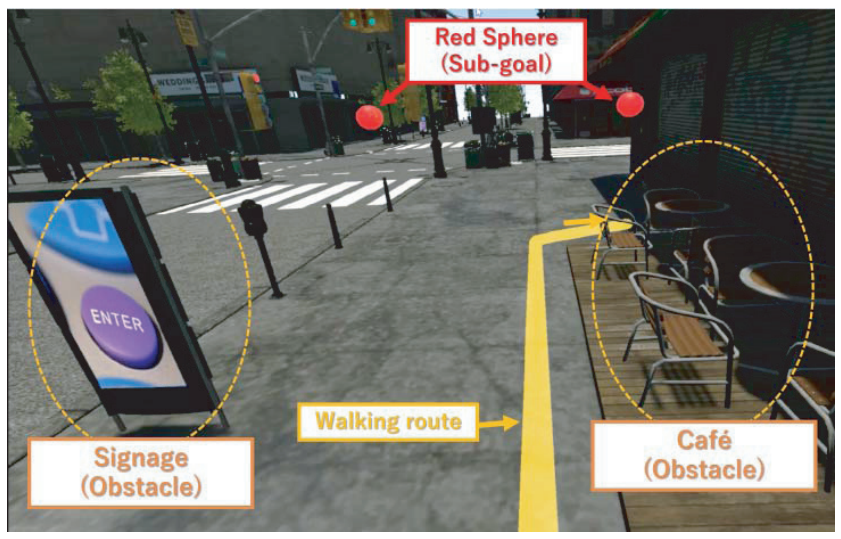

Fig. 6 Obstacles and sub-goals within a computer-generated real environment. Note that texts, bounding boxes, and the broken lines are not indicated in the actual HMD display.

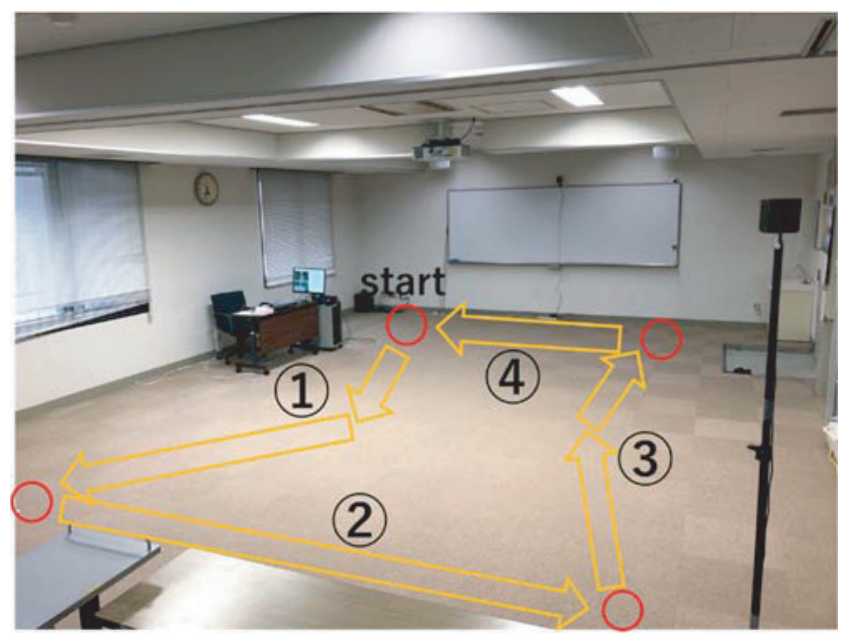

Fig. 7 The experimental environment. The orange arrows represent the optimal walking route. The red circles represent sub-goals and are presented as the red spheres within the HMD display.

field of view with appropriate distance.

\subsection{Experimental Task}

In the experimental task, each subject walked along the indicated counterclockwise route through the sub-goals highlighted by the red spheres in Fig. 3 while avoiding obstacles such as a café and a display, as depicted in Fig. 6. As soon as a subject entered a certain vicinity of a sub-goal, a new red sphere was automatically displayed along with the walking route to the next sub-goal (Figs. 3 and 6). When a subject returned to his starting position, the experimental task was considered to have been completed. We conducted this experiment within a $6 \mathrm{~m} \times 8 \mathrm{~m}$ area, as depicted in Fig. 7, which was completely free from obstacles. The red spheres displayed on the sidewalk in Fig. 3 corresponded to the red circles depicted in Fig. 7. The subjects walked along the indicated routes while constantly looking at the character presented within the VR live content. 


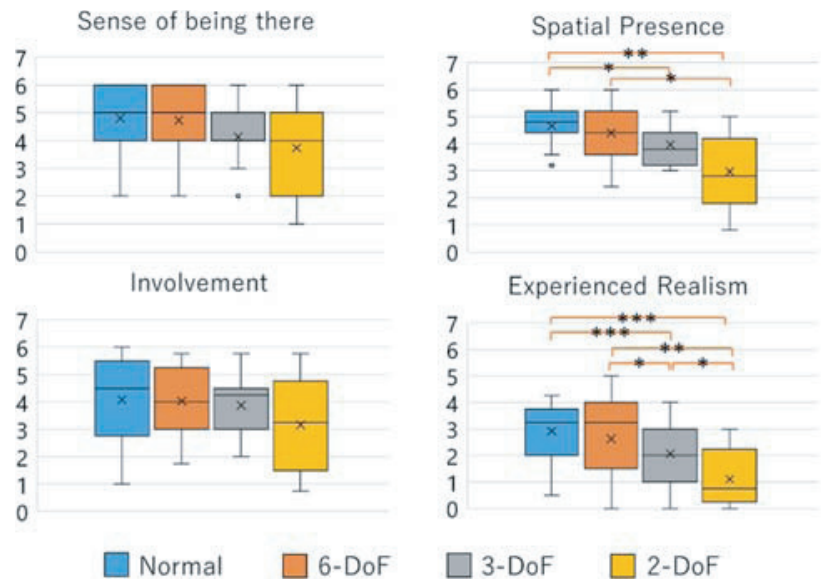

Fig. 8 Result obtained via IPQ.

\subsection{Methods of Evaluation}

To answer RQ1, we focused on the 6-DoF condition, which is a natural preference among users experiencing play roomscale VR, and evaluated the users' VR immersion and sense of security while executing the experimental tasks. VR immersion was evaluated using the Igroup Presence Questionnaire (IPQ) and a user questionnaire [31]. On the other hand, the users' sense of security was evaluated on the basis of the difference between the trajectories of the displayed walking route and the route actually taken by each subject. To answer RQ2, we compared these data corresponding to each DoF condition.

\subsection{Result}

We obtained the following experimental results by performing the aforementioned experiment on 15 participants (14 male, 1 female, each within the age range of 22-25 years). Estimates of the subjects' immersion obtained via the IPQ have been depicted in Fig. 8. We conducted the Wilcoxon signed-rank test to determine the differences between the distributions of scores of the evaluated items. In Fig. 8, the orange square brackets denote significant differences $(* \mathrm{p}<$ $0.05, * * \mathrm{p}<0.01, * * * \mathrm{p}<0.001)$. We used Bonferroni correction to adjust the $\mathrm{p}$-values. In terms of experienced realism of IPQ, there was a significant difference between "Normal and 3-DoF ( $p<0.001, r=-0.85)$ ", "Normal and 2-DoF ( $\mathrm{p}<0.001, \mathrm{r}=-0.86)$ ", "6-DoF and 2-DoF $(\mathrm{p}<0.01, \mathrm{r}=$ $-0.77)$ ", "6-DoF and 3-DoF ( $<<0.05, \mathrm{r}=-0.62)$ ", and "3DoF and 2-DoF ( $p<0.05, r=-0.72)$ ". Next, in terms of spatial presence of IPQ, there was a significant difference between "Normal and 2-DoF ( $p<0.01, r=-0.79)$ ", "Normal and 3-DoF ( $\mathrm{p}<0.05, \mathrm{r}=-0.69)$ ", and "6-DoF and 2DoF ( $\mathrm{p}<0.05, \mathrm{r}=-0.80$ )". However, we could not find a significant difference regarding the sense of being there and involvement of the IPQ. In the 2-DoF condition, the spatial presence and experienced realism are reduced compared to any other conditions. In the 6-DoF condition, any elements of the IPQ can maintain scores compared to the Normal con-
Table 1 Experimental data.

\begin{tabular}{l|ccc}
\hline \hline & 6-DoF & 3-DoF & 2-DoF \\
\hline Task end time (s) & $52.2(16.7)$ & $51.1(13.5)$ & $51.8(12.8)$ \\
Total walking distance (m) & $20.6(0.87)$ & $20.4(0.78)$ & $20.4(0.83)$ \\
Gap between route presented and subject actually walked $(\mathrm{m})$ & $3.46(0.75)$ & $3.14(0.73)$ & $3.29(0.40)$ \\
Character view time (s) & $0.77(0.08)$ & $0.71(0.11)$ & $0.88(0.07)$ \\
Foot confirmation time (s) & $0.074(0.049)$ & $0.079(0.061)$ & $0.038(0.050)$ \\
Head rotation yaw (degrec/s) & $20.8(5.13)$ & $18.6(3.14)$ & $18.8(3.10)$ \\
Head rotation pitch (degree/s) & $16.4(2.94)$ & $15.8(3.11)$ & $15.6(3.76)$ \\
Head rotation roll (degree/s) & $8.88(1.91)$ & $8.42(2.06)$ & $8.01(1.51)$ \\
Viewpoint / gaze direction reset (times) & $14.6(6.13)$ & $8.13(4.98)$ & $-(-)$ \\
\hline
\end{tabular}

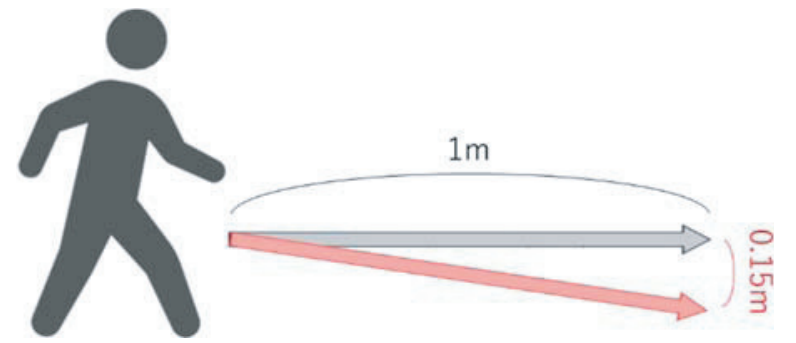

Fig. 9 Deviation between the indicated route and that actually taken by the subject.

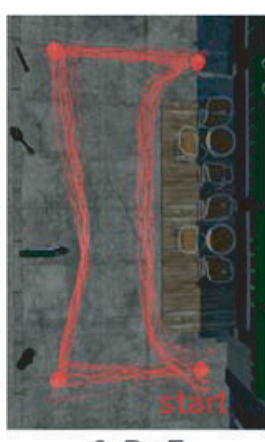

6-DoF

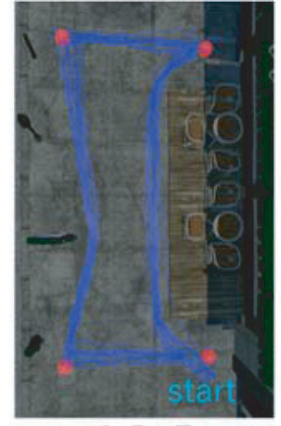

3-DoF

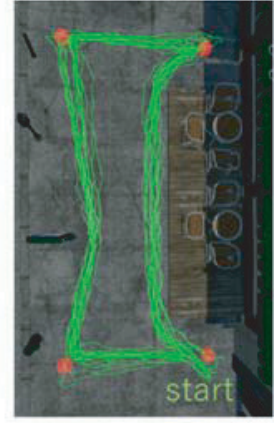

2-DoF
Fig. 10 Routes actually taken by the subjects.

dition.

The results of the experiment have been presented in Table 1. The values in the table denote the averages of the subjects' task data, and the values within brackets represent the associated standard deviations. "Difference between the trajectories of the indicated route and that actually taken by the subject" was measured by adding the lengths of the perpendiculars $(\mathrm{m})$ drawn from the subject's coordinate to the walking route vector displayed to the subject during the previous second. If the total walking distance and the accumulated gap were assumed to be $20 \mathrm{~m}$ and $3 \mathrm{~m}$ approximately, the subject was ascertained to be approximately $0.15 \mathrm{~m}$ away from the indicated route during a walk lasting $1 \mathrm{~m}$ (Fig. 9). The routes actually taken by the subjects have been depicted in Fig. 10. Under all conditions, it was verified that the subjects were able to walk steadily without colliding with obstacles. We did not use eye-tracking devices to measure "Character View Time" and "Foot Confirmation Time". Instead, we simply measured the time for which the HMDs were oriented in the direction of the VR character or at the users' feet. Owing to the lack of eye tracker system, the time that the participant's head was turned in the 
Table 2 User questionnaire

\begin{tabular}{l|ccc}
\hline \hline & 6-DoF & 3 -DoF & 2-DoF \\
\hline Walked while concentrating on VR content & $4.53(1.36)$ & $4.67(0.72)$ & $3.47(1.72)$ \\
Easy to catch character in sight & $4.27(1.94)$ & $5.07(0.70)$ & $5.07(1.53)$ \\
Wallecd in sidewalk without fear & $3.67(1.50)$ & $3.47(1.64)$ & $2.53(1.96)$ \\
Did not feel discomfort to mosement of virtual space when walking & $5.27(0.80)$ & $2.80(1.52)$ & $1.07(1.53)$ \\
\hline
\end{tabular}

direction of the character was considered, intead of the gaze dwell time, although we believe the it is likely that the gaze was turned. As a result, the measurements might not be entirely accurate. However, they can still be used as reasonable references. As the character was always displayed within the subject's view under the 2-DoF condition, the gaze direction reset function could not be used in this case. Based on the Wilcoxon signed rank test performed on each item, we confirmed that "Character View Time" exhibited significant differences $(\mathrm{p}<0.05)$ corresponding to all possible pairs of DoF conditions, while "Foot Confirmation Time" exhibited significant differences $(\mathrm{p}<0.05)$ between the 2DoF and 6-DoF conditions and between the 2-DoF and 3DoF conditions. Further, we used paired t-tests to estimate that "Viewpoint/Gaze Direction Reset" exhibited significant differences $(\mathrm{p}<0.001)$ between the 6-DoF and 3-DoF conditions.

Finally, the results of the user questionnaire have been depicted in Table 2. The values corresponding to the four items denote the averages of the measurements corresponding to the subjects recorded at equal intervals of 7 scales ( 0 6) after each method, and the values in parentheses denote the standard deviations. "Walked While Concentrating on VR Content" was confirmed to exhibit a significant difference $(\mathrm{p}<0.05)$ between the 3-DoF condition and the 2-DoF via the Wilcoxon signed rank test. Using the same method, we confirmed significant differences $(p<0.05)$ between all pairs of DoF conditions with respect to the item "Did Not Feel Discomfort in Movement in Virtual Space While Walking".

Figure 11 (a) depicts a ranking of the subjects' preferences for different DoF conditions. For example, the value corresponding to the entry, 6-3-2, denotes the percentage of subjects who designated their order preference list to be "6DoF, 3-DoF, 2-DoF." Even though the majority of subjects preferred the 6-DoF condition, 3-DoF also exhibited a significant share. Most subjects who preferred the 6-DoF condition opined that "Because I did not feel VR sickness while walking, I could concentrate on the VR content without being distracted by walking." Meanwhile, subjects who preferred the 3-DoF condition opined "Under the 6-DoF condition, it was annoying to have to reset the viewpoint position each time I approached the character." Further, the subjects also opined "Under the 3-DoF condition, I had to focus only on the direction as I was able to maintain a constant distance from the character at all times. Hence, the experience was not annoying at all." Almost all participants feel a large directional gap, which makes them feel VR sickness and uncomfortable in the 2-DOF condition. Based on their responses to the post-experiment questionnaires, subjects were classified into two categories-people (a)

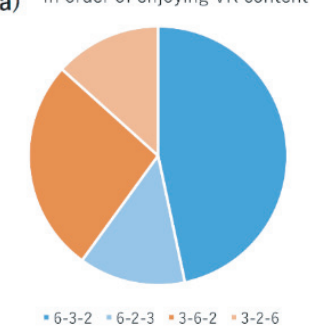

(c) Should system calculate walking route that avoids objects in VR content?

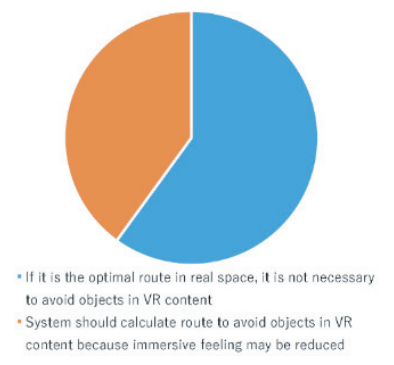

(b)

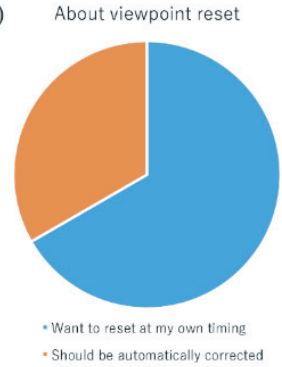

(d) When walking to destination

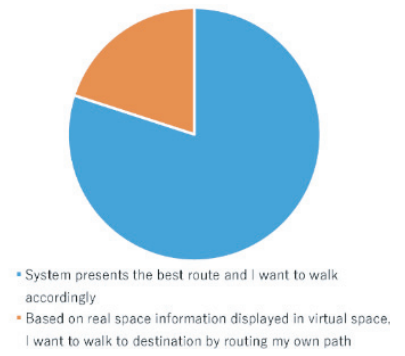

(e)

When assuming that safety in real space is guaranteed surely if you move according to guidance of system, what situation do you want to use VR? (Multiple answers)

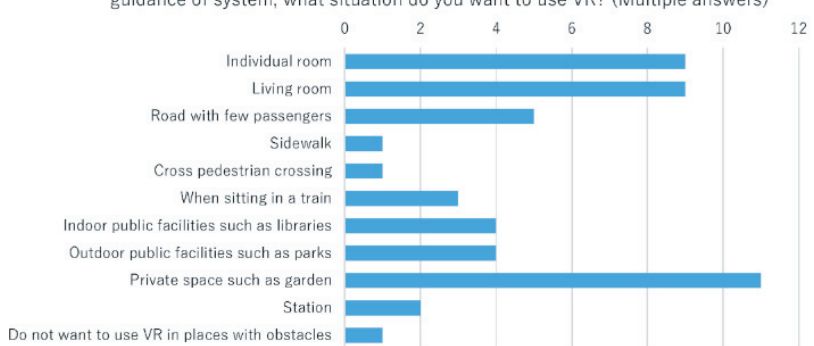

(f) What kind of VR content do you want to use in the above situations? (Multiple answers)

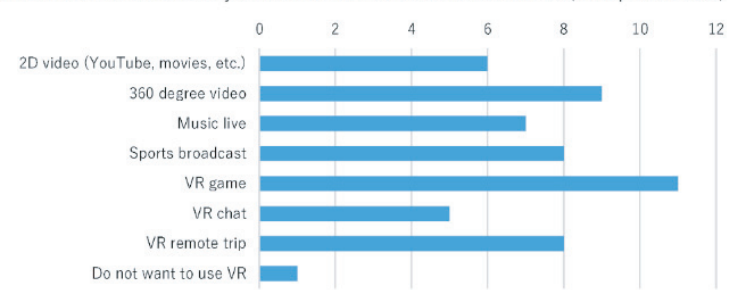

Fig. 11 Result of post-questionnaire.

who emphasized that they did not experience any discomfort while walking and people who emphasized that they did not find resetting the gaze direction or viewpoint annoying. Figure 11 (b) depicts the distribution of subjects into two groups - those who prefer to consciously reset the viewpoint or gaze direction and those who prefer the system to be corrected automatically. Most of the subjects who preferred 3-DoF fell in the latter category. Figure 11 (c) depicts the distribution of subjects with respect to the preference for the system to calculate a route that avoids not only the obstacles in real space but also objects in the virtual space (e.g., characters and stages). Most subjects opined "I want the system to prioritize the safety of the route in real space rather than focus on an immersive VR experience." Figure 11 (d) depicts the distribution of subjects based on their preference of one of the following factors over the other. 
1) A method in which the system calculates the walking route and the user follows it, as in the case of the proposed system.

2) A method in which the user decides the route based on the real-space information displayed within the virtual space, as in the case of the point cloud method and the VR object method [1].

The results indicate that the majority of the subjects preferred walking without being conscious of the real space, instead preferring the system to calculate a safe route, as in the case of automatic driving assistance systems. Figures 11 (e) and 11 (f) depict the distributions of subjects with respect to "the situations where subjects want to use VR" and "the types of content the user wants to experience in those situations," respectively. Many subjects were observed to prefer using VR in the presence of obstacles within a real environment, provided the system was capable of guiding them to their destinations safely. Further, the majority of the subjects preferred static environments where dynamic objects, such as pedestrians and automobiles, did not exist. Further, besides the optimal walking route, some subjects also exhibited an interest in the display of dangerous objects, such as moving pedestrians, traffic lights, and cars, within the virtual environment, as well as a bird's-eyeview map depicting the subjects' progress towards the destination.

\section{Experiments in Dynamic Situations}

The experiments reported in the previous section demonstrated that, under static conditions, the proposed system was capable of safely guiding users along a walking route while avoiding collisions with obstacles corresponding to all DoF conditions. However, the introduction of moving pedestrians into the fray necessitates the constant dynamic revision of the walking route. In order to evaluate the capability of the system to perform its task in such a scenario, we considered an experimental set-up involving two pedestrians walking back and forth on the sidewalk, as depicted in Fig. 12. The movement of the two pedestrians is scenariobased. Two pedestrians are present in the experimental environment, moving back and forth in a rectangular experimental environment at a constant speed in the direction to the long side of the rectangle of the experimental environment. A collision detection between the pedestrian and the subject uses Unity's Collider component. Henceforth, we refer to this scenario as the dynamic situation and the scenario of the previous experiment, which was devoid of moving pedestrians, as the static situation.

\subsection{Experimental Design}

In the previous experiment, we concluded that the user's VR immersion was significantly compromised under the 2-DoF condition. Hence, we restricted the dynamic situation-based experiment to only the 3-DoF and 6-DoF conditions.

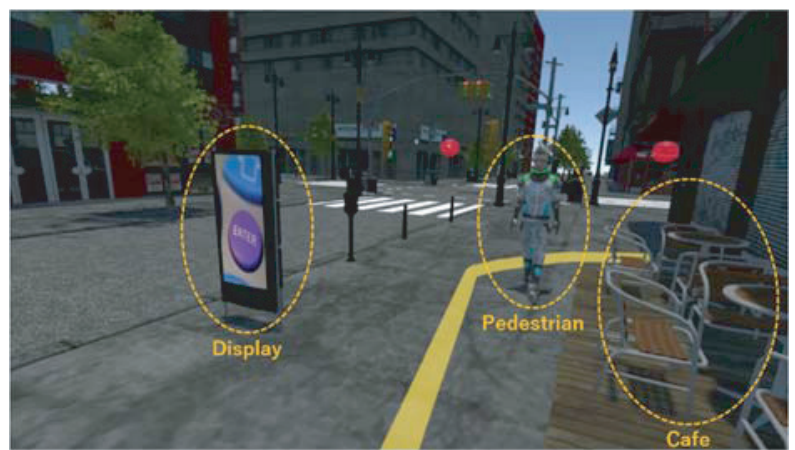

Fig. 12 Obstacles in the dynamic situation.

First, to acquire the reference data for walking, each subject was made to traverse the identical route, as in the previous experiment, using 3-DoF and 6-DoF conditions in the dynamic situation while paying attention solely to pedestrians in the absence of any VR content (base condition). Then, the subjects were made to traverse the route while experiencing VR content using 3-DoF and 6-DoF conditions in the static condition. Subsequently, the subjects were made to traverse the route while experiencing VR content in 3DoF and 6-DoF conditions in the dynamic situation. Thus, the subjects were made to traverse the route twice, without any interruption or rest between static and dynamic situations. The reason for designing the task to be performed consecutively without a break or interruption was that the removal of the HMD, which occurred at each interruption or rest, was a significant burden for both the subject and the experimenter. 3-DoF and 6-DoF were adopted at random to eliminate any residual effect from their ordering. Subjects walked along the route while experiencing VR content by relying on the indicated walking route that was observed to fluctuate in real time in the dynamic situation.

After completing the experimental tasks, the subjects were asked to watch a video shot from a firstperson perspective depicting their own movement within the computer-generated environment.

\subsection{Results}

We performed the aforementioned experiment on 13 participants (12 males, 1 female, each within 22-27 years old) Nine subjects joined the experiment mentioned in Section 4. The results of the subjects' VR immersion obtained via the IPQ have been depicted in Fig. 13. We conducted twoway repeated measures ANOVA to analyze the associated distributions of scores. In Fig. 13, the orange square brackets indicate significant differences $\left(* \ldots \mathrm{p}<0.05,{ }^{* *} \ldots \mathrm{p}<\right.$ 0.01). We used Bonferroni correction to adjust the p-values. The items "Spatial Presence" and "Involvement," were confirmed to exhibit significant differences * between the DoF conditions (3-DoF and 6-DoF). Further "Experienced Realism" exhibited a significant difference $* *$ between the two environmental situations (static and dynamic). In terms of involvement of the IPQ, both the 3-Dynamic and 6-Dynamic 

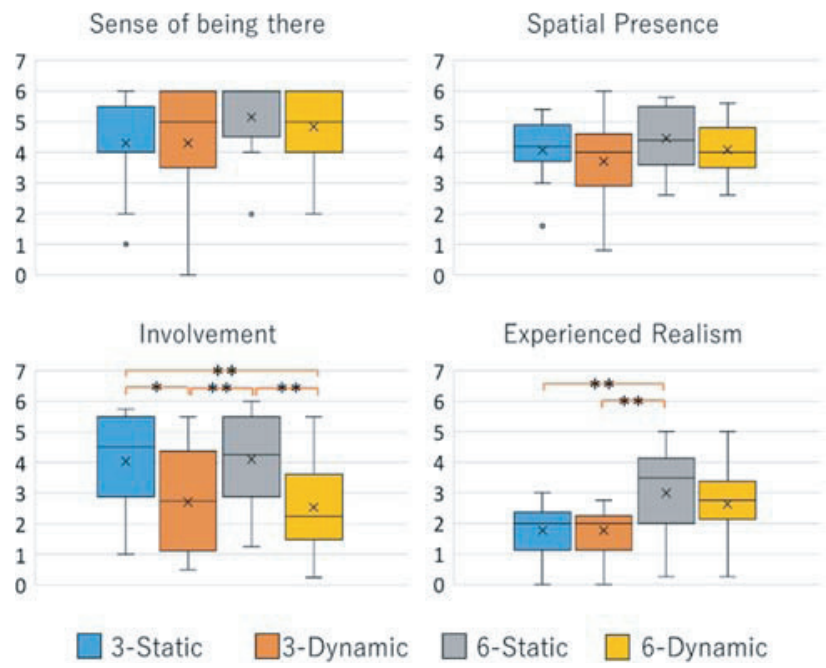

Fig. 13 Result of IPQ (Dynamic situation experiment).

Table 3 Experimental data (Dynamic situation experiment).

\begin{tabular}{|c|c|c|c|c|c|c|}
\hline & Base-Stabe & Base-Dynamic & 3-Static & 3-Dynamic & 6-Stasc & 6-Dynamic \\
\hline Tadk and time (s) & $322(6.13)$ & $33.3(8.45)$ & $398(877)$ & $403(9,31)$ & $428(9.87)$ & $43.7(10.2)$ \\
\hline Total walking distance (m) & $195(0.53)$ & $199(0.97)$ & $19.7(0 \mathrm{~m})$ & $20.1(0.85)$ & $20.0(0.85)$ & $20.8(1.15)$ \\
\hline $\begin{array}{l}\text { Gap between route presented and } \\
\text { subject axtually walked (m) }\end{array}$ & $2.60(0.39)$ & $3.40(0.76)$ & $284(0.66)$ & $3.77(1.07)$ & $3.02(0.84)$ & $4.01(0.79)$ \\
\hline Character view time (s) & $\cdot(-)$ & $\cdot(\cdot)$ & $0.680099)$ & $0.50(0.10)$ & $0.77(0.08)$ & $0.63(0.12)$ \\
\hline Fon coefirmation time (s) & $0.17(0.11)$ & $0.25(0.16)$ & $0.10(0.10)$ & $0.18(0.14)$ & $0.08(0.07)$ & $0.16(0.13)$ \\
\hline Head rotation yaw (degree / s) & $27.7(5.20)$ & $33,4(9.35)$ & $21.4(430)$ & $255(497)$ & $20.7(3.25)$ & $25.2(4.29)$ \\
\hline Head rotation pitch (degree / s) & $18.9(4.43)$ & $18.5(4.41)$ & $15.4(4.36)$ & $172(4.23)$ & $15,4(4.31)$ & $17.0(4.62)$ \\
\hline Head rotatice foll (degrece / $\mathrm{s}$ ) & $9.93(1.20)$ & $10.6(1.50)$ & $805(1,42)$ & $904(1.70)$ & $8.07(1.60)$ & $8.66(1.97)$ \\
\hline $\begin{array}{l}\text { Vieapoint / yare dirextion roset } \\
\text { (times) }\end{array}$ & $\cdot(-)$ & $-(\rightarrow)$ & $8.23(3.50)$ & 105 (4.98) & $152(4.80)$ & $18.6(4.50)$ \\
\hline Collision itimest & $0(0)$ & 0080287 & $0 \cos (078)$ & $1.77(0.73)$ & $008028)$ & $\{85(090)$ \\
\hline
\end{tabular}

conditions reduced scores compared to static conditions. In addition, in terms of experienced realism, scores of 3-Static and 3-Dynamic conditions reduced scores compared to the 6-Static and 6-Dynamic conditions.

The detailed experimental statistics have been depicted in Table 3. The values denote the averages of each subject's task data, and the values within brackets denote the associated standard deviations. We conducted two-way repeated measures ANOVA on four items, excluding base conditions, to analyze the distributions of scores. The items, "Task end time," "Total walking distance," "Character view time," and "Viewpoint / gaze direction reset," exhibited significant differences $(\mathrm{p}<0.01)$ between the two DoF conditions. Further, all items excluding "Task end time" exhibited a significant difference $(\mathrm{p}<0.01)$ between the two environmental situations (static and dynamic).

Finally, we present the results of the post-experimental questionnaire. In response to the question, "Do you think 6-DoF or 3-DoF is more appropriate when experiencing VR content while walking to a destination in real space?", 54 percent of the subjects opted for the 3-DoF condition. In response to the question, "Based on the first-person video, do you think that you were able to safely traverse a route in the simulated environment?", 85 percent of the subjects answered in the negative. Further, the following comments were made by the subjects: "I felt difficulty in dealing with moving objects," "I think the sense of security would be improved if the approaching directions of moving objects, such as cars and pedestrians, were conveyed to me," "I was surprised that I was walking more naturally than I had realized. I was wondering whether I was acting suspiciously. I did not experience much of a difference even in the absence of pedestrians," "I was walking more haltingly than I had realized. I feel that the dynamic changes to the route need to be conveyed a little earlier'. In response to the question, "Were you able to predict the movement of the pedestrian based on the fluctuations of the walking route?", 69 percent of the subjects answered in the negative. All the subjects felt that the real-space information should be displayed within the virtual environment, rather than just the walking route, in the dynamic situation. Examples of real-space information that subjects wanted to be displayed included the coordinates and direction of moving objects, and an alert to indicate an immediate halt.

\section{Discussion}

We begin by discussing RQ1. In terms of VR immersion, no difference was noticed between the 6-DoF condition in the static experiment and the normal scenario, which involves the experience of VR without walking. Therefore, it can be concluded that indicating the optimal walking route within the VR environment successfully conveys real-space information to the VR-user without compromising VR immersion in the static situation. However, in the dynamic situation, the user's VR immersion was compromised to a significant extent compared to the static situation. We conjecture that if real-space information were presented in a manner that allows the user to walk without any apprehension, then the user will be able to experience VR content while walking in a dynamic environment without compromising VR immersion. It is evident from Fig. 10 that users were able to walk steadily without colliding with obstacles while experiencing VR content in the static situation. In addition, it is evident from Fig. 11 (d) that users preferred route guidance by the system to the direct presentation of real-space information on the HMD. However, in the dynamic situation, as recorded in Table 3, subjects were observed to collide with the virtual pedestrians several times. In this scenario, the user was presented with the optimal route at every moment. However, in the time that it took the subject to actually walk along the route, the optimal route had changed already. Thus, to resolve this issue, it is necessary to consider an algorithm that incorporates motion prediction to calculate predictive optimal route corresponding to the moment when the user is actually going to walk. Further, as the experimental results were based on the assumption that the system was capable of calculating the optimal route, appropriate technology to accurately sense the real environment around the user is also necessary.

Next, we discuss RQ2. In general, users' VR immer- 
sion was observed to decay with a decrease in the degrees of freedom. In particular, discomfort during walking was observed to greatly affect VR immersion. However, discomfort while walking was observed to be more pronounced corresponding to the 6-DoF condition because users found it easier to concentrate on the VR content under the 3-DoF condition. In contrast, the relative changes in position between the user and character in the 6-DoF condition induced by walking adversely affected the concentration of the user on the VR content. Although it was very easy for users to maintain the VR character within their viewing range in the 2-DoF condition, concentration upon the VR content was more difficult because of the discomfort caused by movement in the virtual space.

The statistics related to "Task end time," "Total walking distance," and "Route subjects actually walked" indicated that despite experiencing discomfort while walking, users were still able to walk steadily. Based on a particular subject's opinion, we expect the adverse effects of high degrees of freedom on the ease of walking to be reduced by familiarization. Although a slightly greater number of users preferred the 6-DoF condition than the 3-DoF condition, as depicted in Fig. 11 (a), users were divided into two groups-people who emphasized that they experienced no discomfort during walking and people who emphasized that they did not find resetting the viewpoint or gaze direction annoying.

In the dynamic situation, as subjects were required to traverse the route two laps within one task (the first lap was in the static condition and the second lap was in the dynamic condition) within one task, the walking path was longer than in the previous experiment. This increased the proportion of users who preferred 3-DoF in this case. Based on this result, we conclude that there is a possibility that users will get used to the discomfort during walking and 3-DoF will become the preferred DoF condition as walking routes become longer.

Next, we discuss the performance of the proposed method in static and dynamic situations. On investigating the situations, we found that many users preferred using the system in an environment with few high-risk objects whose coordinates change in real time, such as pedestrians and cars. In the dynamic situation, we found that the proposed walking route presentation algorithm was not capable of safely guiding users to their destinations. Further, it is necessary to investigate whether a user can safely reach the destination by walking along the route and can walk without fear even in a situation containing moving objects. Further, although VR content is user-dependent, the optimal DoF condition may depend on the type of VR content used. For example, 3D VR content usually involves a representation of the surface of the ground as used in this experiment. In such cases, the users experienced discomfort when the coordinates of their avatar did not change while walking under the 3-DoF condition. On the other hand, within 2D video content, such as watching a video from the user's view that does not require a representation of a ground surface, users did not experience any discomfort. Based on the results, it seems that the consciousness of one's feet and the concept of a ground surface are strongly correlated to the discomfort experienced by the subjects. Compared to our proposed visited obstacle method [1], [2], the visited obstacle method requires the correspondence information between a VR object and a real-space object beforehand. However, the user using this method can walk without knowing the correspondence.

In this study, we used NavMesh to calculate the optimal walking route in the simulated real environment by searching for neighboring polygons via the $A^{*}$ algorithm, and calculating the shortest route to the destination. However, NavMesh cannot be used in real world. We need some automatic calculation of the optimal walking route instead of NavMesh. To realize this, a possible alternative method is described below. The automatic calculation of the optimal walking route based on the information of the user's real environment necessitates the generation of a map via simultaneous localization and mapping (SLAM) [27], which is used to represent real spaces via 3D reconstruction technology. After generating the map, the walking route can be calculated by executing the following steps. A method proposed by Lee et al. [28] was used to create a grid map using 3D point cloud data and search for the optimal route within the walkable region. The direction in which the user should walk is predicted based on a pedestrian model, such as the social force model for pedestrian dynamics [29], [30], after determining the coordinates of all obstacles and pedestrians via clustering of the $3 \mathrm{D}$ data.

Further, the proposed system was similar to an automobile driving assistance system. While using this system, the user only followed the walking route, without the awareness of other pedestrians around him. Thus, depending on the parameters required to calculate the walking route, there is a possibility for the system to generate a dangerous route where the user is likely to collide with other pedestrians. Moreover, when the other pedestrians see a user walking wearing an HMD, they may not approach the HMD user as they are unlikely to be able to predict the route to be taken by the HMD user. Therefore, it is necessary to investigate the social impact of this system.

\section{Conclusion}

In this paper, we proposed a navigation system capable of automatically calculating the optimal walking route for a VR-user that avoids collisions with surrounding obstacles and preserves the VR immersion of the user. We simulated a real environment and conducted experiments to answer the following two questions,

1) Was the system capable of safely guiding users to their destinations in real space without compromising their VR immersion by displaying only the optimal walking route within the VR environment?.

2) How many degrees of freedom are required to track the user's head to ensure optimal VR immersion while guid- 
ing the user toward the destination?

The experimental results demonstrated that, in situations where the system could calculate a safe route, it was capable of safely guiding users to their destinations without compromising their VR immersion in a static situation. In addition, we found that users' preferences were greatly divided between 6-DoF and 3-DoF when using VR while walking in real space. However, we expect that users will prefer 3-DoF conditions when they have to walk over longer distances while experiencing VR content.

To safely guide a user to a destination in a dynamic situation, it was necessary to calculate the walking route using moving body predictions and to display the moving body within the virtual space in order to preserve VR immersion for the user. Finally, in this experiment, collisions occurred in many cases despite the absence of cars, rugged terrain and a considerably less number of pedestrians. In real environments, especially in urban areas, it is easy to imagine a more dangerous situation for VR Zombie. Therefore, in terms of ethics, we do not recommend experiencing VR content in places with pedestrians and moving cars.

\section{References}

[1] K. Kanamori, N. Sakata, T. Tominaga, Y. Hijikata, K. Harada, and K. Kiyokawa, "Obstacle avoidance method in real space for virtual reality immersion," Proc. the IEEE International Symposium for Mixed and Augmented Reality 2018, pp.80-89, 2018.

[2] K. Kanamori, N. Sakata, T. Tominaga, Y. Hijikata, K. Kiyokawa, and K. Harada, "Walking support in real space using social force model when wearing immersive hmd" Adjunct Proc. IEEE International Symposium for Mixed and Augmented Reality, pp.250-253, 2018.

[3] E.M. Kolasinski, Simulator Sickness in Virtual Environments (no.ARI-TR-1027), Army Research Inst for the Behavioral and Social Sciences Alexandria Va, 1995.

[4] J.J. LaViola Jr, "A discussion of cybersickness in virtual environments," ACM SIGCHI Bulletin, vol.32, no.1, pp.47-56, 2000.

[5] F. Steinicke, G. Bruder, K. Rothaus, and K. Hinrichs, "Poster: A virtual body for augmented virtuality by chroma-keying of egocentric videos," In IEEE Symposium on 3D User Interfaces, pp.125-126, 2009.

[6] F. Tecchia, G. Avveduto, R. Brondi, M. Carrozzino, M. Bergamasco, and L. Alem, "I'm in VR!: using your own hands in a fully immersive MR system," Proc. the 20th ACM Symposium on Virtual Reality Software and Technology, ACM, pp.73-76, 2014.

[7] M. McGill, D. Boland, R. Murray-Smith, and S. Brewster, "A dose of reality: Overcoming usability challenges in vr head-mounted displays," Proc. the 33rd Annual ACM Conference on Human Factors in Computing Systems, pp.2143-2152, 2015.

[8] P. Budhiraja, R. Sodhi, B. Jones, K. Karsch, B. Bailey, and D. Forsyth, "Where's My Drink? Enabling Peripheral Real World Interactions While Using HMDs" arXiv preprint arXiv:1502.04744, 2015.

[9] J. Fung, C.L. Richards, F. Malouin, B.J. McFadyen, and A. Lamontagne, "A treadmill and motion coupled virtual reality system for gait training post-stroke," CyberPsychology \& behavior, vol.9, no.2, pp.157-162, 2006.

[10] M. Nabiyouni, A. Saktheeswaran, D.A. Bowman, and A. Karanth, "Comparing the performance of natural, semi-natural, and non-natural locomotion techniques in virtual reality," Proc. IEEE Symposium on 3D User Interfaces (3DUI), pp.3-10, 2015.
[11] S. Tregillus and E. Folmer, "Vr-step: Walking-in-place using inertial sensing for hands free navigation in mobile vr environments," Proc. the 2016 CHI Conference on Human Factors in Computing Systems, pp.1250-1255, 2016.

[12] M. Usoh, K. Arthur, M.C. Whitton, R. Bastos, A. Steed, M. Slater, and F.P. Brooks, "Walking > walking-in-place > flying, in virtual environments," Proc. the 26th annual conference on Computer graphics and interactive techniques. Addison-Wesley/ACM Press, pp.359-364, 1999.

[13] P.T. Wilson, W. Kalescky, A. MacLaughlin, and B. Williams, "VR locomotion: walking $>$ walking in place $>$ arm swinging," Proc. the 15th ACM SIGGRAPH Conference on Virtual-Reality Continuum and Its Applications in Industry-Volume 1, pp.243-249, 2016.

[14] K.H. Sing and W. Xie, "Garden: A Mixed Reality Experience Combining Virtual Reality and 3D Reconstruction," Proc. the 2016 CHI Conference Extended Abstracts on Human Factors in Computing Systems, pp.180-183, 2016.

[15] E.J. Arthur, P.A. Hancock, and S.T. Chrysler, "The perception of spatial layout in real and virtual worlds," Ergonomics, vol.40, no.1, pp.69-77, 1997.

[16] C.S. Sahm, S.H. Creem-Regehr, W.B. Thompson, and P. Willemsen, "Throwing versus walking as indicators of distance perception in similar real and virtual environments," ACM Transactions on Applied Perception (TAP), vol.2, no.1, pp.35-45, 2005.

[17] J.M. Knapp and J.M. Loomis, "Limited field of view of headmounted displays is not the cause of distance underestimation in virtual environments," Presence: Teleoperators Virtual Environ, vol.13, no.5, pp.572-577, 2004.

[18] R. Messing and F.H. Durgin, "Distance perception and the visual horizon in head-mounted displays," ACM Transactions on Applied Perception (TAP), vol.2, no.3, pp.234-250, 2005.

[19] A.R. Richardson and D. Waller, "Interaction with an immersive virtual environment corrects users' distance estimates," Human Factors, vol.49, no.3, pp.507-517, 2007.

[20] S.A. Linkenauger, B.J. Mohler, and H.H. Bülthoff, "Welcome to wonderland: The apparent size of the self-avatar hands and arms influences perceived size and shape in virtual environments," Journal of Vision, vol.11, no.11, 2011.

[21] S.A. Linkenauger, B.J. Mohler, and D.R. Proffitt, "Body-Based Perceptual Rescaling Revealed through the Size - Weight Illusion," Perception, vol.40, no.10, pp.1251-1253, 2011.

[22] P. Rahimian, E.E. O’Neal, J.P. Yon, L. Franzen, Y. Jiang, J.M. Plumert, and J.K. Kearney, "Using a virtual environment to study the impact of sending traffic alerts to texting pedestrians," Proc. IEEE Virtual Reality 2016 (VR2016), pp.141-149, 2016.

[23] U. Rehman and S. Cao, "Augmented-reality-based indoor navigation: A comparative analysis of handheld devices versus google glass," IEEE Trans. Human Machine Systems, vol.47, no.1, pp.140-151, 2017.

[24] H.N. Sharma, Z.O. Toups, I. Dolgov, A. Kerne, and A. Jain, "Evaluating display modalities using a mixed reality game," Proc. the 2016 Annual Symposium on Computer-Human Interaction in Play, pp.65-77, 2016.

[25] F. Buttussi and L. Chittaro, "Effects of different types of virtual reality display on presence and learning in a safety training scenario," IEEE Trans. Vis. Comput. Graphics, vol.24, no.2, pp.1063-1076, 2018.

[26] S.K. Pal, M. Khan, and R.P. McMahan, "The benefits of rotational head tracking," Proc. the 2016 3D User Interfaces (3DUI) 2016, pp.31-38, 2016.

[27] T. Bailey and H. Durrant-Whyte, "Simultaneous localization and mapping (slam): Part ii," IEEE Robotics \& Automation Magazine, vol.13, no.3, pp.108-117, 2006

[28] Y.H. Lee and G. Medioni, "Rgb-d camera based wearable navigation system for the visually impaired," Computer Vision and Image Understanding, vol.149, pp.3-20, 2016.

[29] D. Helbing and P. Molnar, "Social force model for pedestrian dy- 
namics," Physical review E, vol.51, no.5, pp.4282-4286, 1995.

[30] D. Helbing, I. Farkas, and T. Vicsek, "Simulating dynamical features of escape panic," Nature, vol.407, no.6803, pp.487-490, 2000.

[31] T. Schubert, F. Friedmann, and H. Regenbrecht, "The experience of presence: Factor analytic insights," Presence: Teleoperators \& Virtual Environments, vol.10, no.3, pp.266-281, 2001.

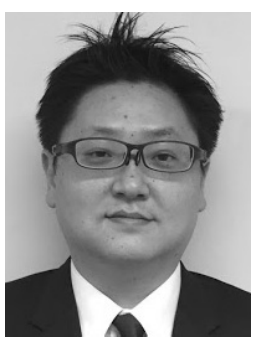

Nobuchika Sakata graduated from department of Institute of Engineering Mechanics, University of Tsukuba in 2002. He completed doctoral program of department of Institute of Engineering Mechanics, University of Tsukuba in 2007. He is a associate professor with the Nara Institute of Science and Technology, since 2018. He has Ph.D. in Engineering.

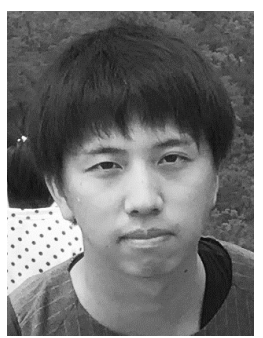

Kohei Kanamori graduated from Engineering Science Department of Osaka University in 2019.

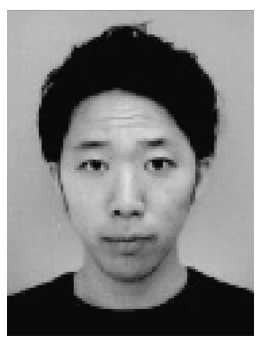

Tomu Tominaga graduated from Engineering Science Department of Osaka University in 2014. He completed doctoral program of department of Institute of Engineering Science Department of Osaka University in 2019. He has Ph.D. in Engineering.

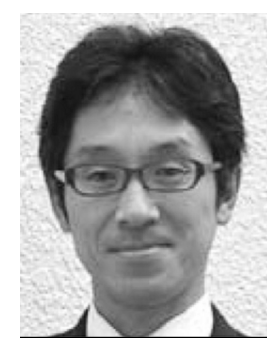

Yoshinori Hijikata graduated the master course of Osaka University in engineering science in 1998. After graduation, he joined IBM research, Tokyo research Laboratory. He received Ph.D. in 2002 from Osaka University. He was assistant professor (2002-2006) and associate professor (2007-2017) in Osaka University. He was a visiting researcher in GroupLens Research of University of Minnesota in 2014. Currently, he is a professor in Kwansei Gakuin University.

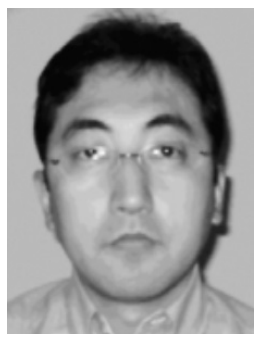

Kensuke Harada received his B.S., M.S., and Doctoral degrees in Mechanical Engineering from Kyoto University in 1992, 1994, and 1997 , respectively. He is a professor with the Osaka University, since 2016. He has Ph.D. in Engineering.

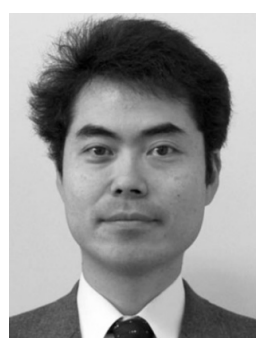

Kiyoshi Kiyokawa received the MS and $\mathrm{PhD}$ degrees in information systems from the Nara Institute of Science and Technology, in 1996 and 1998, respectively. He is a professor with the Nara Institute of Science and Technology, since 2017. He has Ph.D. in Engineering. 